\title{
Incidence and predictors of nevirapine and efavirenz-associated rash among Indonesian HIV patients
}

\author{
Maryatun Hasan, ${ }^{1,2}$ Evy Yunihastuti, ${ }^{2}$ Teguh $H$ Karjadi, ${ }^{2}$ Murdani Abdullah ${ }^{2}$
}

\begin{abstract}
Background: Many drugs used for the treatment of HIV disease can cause drug hypersensitivity reactions. Since 2002, World Health Organization (WHO) has recommended the use of nevirapine and efavirenz as part of first line antiretroviral therapies for several years. Both of the drugs had equivalent clinical efficacy but differences in toxicity profile.
\end{abstract}

Objectives: We aimed to determine the incidence and predictors of nevirapine and efavirenz-associated rash among Indonesian HIV-infected patients.

Methods: A retrospective cohort study was conducted among Indonesian patients who were using nevirapine or efavirenz between January 2004 to December 2013. All eligible predictors were analyzed using bivariate and multivariate analysis.

Results: 2,071 patients received nevirapine and 1,212 received efavirenz as first line therapies. The cumulative incidence of nevirapine-associated rash was $14 \%$ (95\% CI: $12.5-15.5 \%)$ and evafirenz-associated rash was $4.5 \%$ (95\% CI: 3.4$5.8 \%$ ). Severe reactions occurred $1 \%$ patients receiving NVP and $0.1 \%$ patients receiving EFV, but no patients died due to these conditions. Predictors independently related with nevirapine-associated rash were female gender (adjusted RR $=1.622$; 95\% CI: 1.196-2.199; $p=0.002$ ), baseline absolute CD4 count above 200 cells $/ \mathrm{mm}^{3}$ (adjusted RR = 1.387; 95\% CI: 1.041-1.847; $p=0.025$ ), and hepatitis C co-infection (adjusted RR = 1.507; 95\% CI: 1.138-1.995; $p=0.004$ ). Baseline ALT level > 1.25 times upper normal limit (adjusted RR $=1.508 ; 95 \%$ CI: 0.998-2.278; $p=0.051$ ) had a tendency to be a good predictor. None of the risk factors investigated was associated with developing efavirenz-associated rash.

Conclusion: Female, baseline absolute CD4 count above 200 cells $/ \mathrm{mm}^{3}$, hepatitis C co-infection and baseline ALT levels more than 1.25 times upper normal limit were predictors for nevirapine-associated rash in HIV patients.

Key words: HIV, nevirapine, efavirenz, predictor, rash

\section{Citation:}

Hasan, M., Yunihastuti, E., Karjadi, T. H., Abdullah, M. (2022). Incidence and predictors of nevirapine and efavirenz-associated rash among Indonesian HIV patients. Asian Pac J Allergy Immunol, 40(2), 142-147. https://doi.org/10.12932/ap-080719-0596

\footnotetext{
Affiliations:

${ }^{1}$ Department of Internal Medicine, Faculty of Medicine,

Universitas Syiah Kuala/ZainoelAbidin Hospital, Aceh, Indonesia

Department of Internal Medicine, Faculty of Medicine,

Universitas Indonesia/CiptoMangunkusumo Hospital, Jakarta,

Indonesia
}

Corresponding author:

Evy Yunihastuti

Jl Diponegoro 71, Jakarta Pusat, Indonesia, 10320

E-mail: evy.yunihastuti@gmail.com, evy.yuniastuti@ui.ac.id

\section{Introduction}

People living with HIV/AIDS (PLWHA) are generally more likely to experience both drug hypersensitivity and severe drug hypersensitivity reaction compared to general population. Manifestations of these reactions include Stevens-Johnson Syndrome (SJS) and Toxic Epidermal Necrolysis (TEN), due to immune function alteration, drug metabolism change, and polypharmacy among PLWHA., ${ }^{1,2}$

World Health Organization (WHO) recommends a combination of two nucleoside reverse transcriptase inhibitors (NRTIs) and one non-nucleoside reverse transcriptase inhibitor (NNRTI) either nevirapine (NVP) or efavirenz (EFV) as first line antiretroviral therapy (ART) in resource-limited settings, including Indonesia. Both NVP or EFV have equivalent clinical efficacy but different toxicity profile. These drugs have been reported to cause hypersensitivity reactions 
with primary manifestation in the form of skin rashes from mild to potentially life-threatening. ${ }^{2-5}$

The influence of race to NNRTI-related rash was unknown. High incidence of NVP-associated rash in Asian population ranging from $2.6 \%$ to $54.4 \%$ and commonly emerges at 12 days to 6 weeks after initiation. ${ }^{1,45}$ Incidence of skin rash in those receiving efavirenz was reported at $8.2 \%$ to $20 \%$ with mild to moderate skin manifestations. They usually emerge on the 8 days after initiation. ${ }^{6,7}$ The incidence of SJS or TEN in those receiving NVP was $0.7-3.9 \%$, much lower in those receiving EFV (0.1-0.2\%). ${ }^{1,8,9}$

Skin rash caused by either NVP or EFV will influence quality of life and adherence to therapy among PLWHA. Knowing spesific factors that might predict skin rash will help in drug-choosing and finally improving care for PLWHA. In this study, we aim to determine the incidence of NVP and EFV-associated rash and predictors that might associate with it.

\section{Material and Methods}

We conducted a retrospective cohort study of HIV-infected adult patients in HIV Integrated Clinic Cipto Mangunkusumo General Hospital Jakarta, Indonesia. This is a largest HIV and referral center hospital in Indonesia. All patients aged above 18 years old initiating NNRTI-based regimen in this center from January 2004 to December 2013 were included in this study. Patients who used NVP, either as naïve therapy or substitution regimen, were included in NVP group and those who used EFV, either as naïve therapy or substitution regimen, were included in EFV group. The study protocol was approved by the Institutional Review Board Faculty of Medicine, Universitas Indonesia.

The primary outcome was cumulative incidence of NVP or EFV-associated rash. Rash could manifest as diffuse maculopapular or erythematous rash, serum sickness-like reaction, hypersensitivity syndromes, SJS, and TEN. Secondary outcomes were predictors for NVP or EFV-associated rash. Possible baseline predictors included in the analysis were gender, CD4 lymphocyte count, serum transaminase enzyme level, hepatitis co-infection, body weight, and HIV stage. Gradual escalating dose for NVP was also evaluated.

Statistical analysis was performed using Statistical Package for Social Science program version 20.0 (SPSS Inc, Chicago IL, USA). All predictors underwent bivariate analysis using Chi-Square test for categorical data and T-test for continuous data with normal distribution or Mann Whitney test for those with abnormal distribution. Data normality was tested using Kolmogorov-Smirnov test. All variables with $p$ value $<0.25$ in bivariate analysis were included in multivariate analysis using logistic regression. A p-value of less than 0.05 was considered statistically significance. Multivariate analysis results were displayed in 95\% confidence interval (95\% CI) and risk ratio (RR). Incomplete data is resolved by multiple imputation techniques.

\section{Results \\ Subject Characteristics}

A total of 2,071 HIV-infected patients initiating NVPbased regimen and 1,212 patients initiating EFV-based regimen were included in the study, with median age 29.7 (minmax 18-70) years old in NVP group and 30 (min-max 18-62) years old in EFV group, and mostly were male (72.2\% in NVP group and $75.2 \%$ in EFV group). The most common risk factor of HIV infection was heterosexual ( $40 \%$ in NVP group and $41.4 \%$ in EFV group). Most of the patients started NVPbased regimen or EFV-based regimen in the late stage $(53.9 \%$ stage III and $20 \%$ stage IV in NVP group; $59.7 \%$ stage III and $25.2 \%$ stage IV in EFV group). Table 1 shows baseline characteristics of subjects enrolled in this study.

\section{Table 1. Subject Characteristics}

\begin{tabular}{|c|c|c|}
\hline \multirow[b]{2}{*}{ Characteristics } & \multicolumn{2}{|c|}{ NNRTI treatment } \\
\hline & $\begin{array}{c}\text { Nevirapine } \\
n=2,071\end{array}$ & $\begin{array}{c}\text { Efavirenz } \\
\mathrm{n}=\mathbf{1 , 2 1 2}\end{array}$ \\
\hline Age, median (min-max), years & $29.7(18-70)$ & $30(18-62)$ \\
\hline Male gender, n (\%) & $1,495(72.2)$ & $912(75.2)$ \\
\hline Being married, n (\%) & $1,078(52.0)$ & $669(55.2)$ \\
\hline CD4, median (IQR), cells $/ \mathrm{mm}^{3}$ & $70(170)$ & $49(121)$ \\
\hline \multicolumn{3}{|l|}{ HIV stage, n (\%) } \\
\hline Stage I & $314(15.2)$ & $114(9.4)$ \\
\hline Stage II & $226(10.9)$ & $69(5.7)$ \\
\hline Stage III & $1,116(53.9)$ & $723(59.7)$ \\
\hline Stage IV & $415(20.0)$ & $306(25.2)$ \\
\hline \multicolumn{3}{|l|}{ HIV risk factors, $\mathrm{n}(\%)$} \\
\hline Sexual & $828(40.0)$ & $502(41.4)$ \\
\hline IDU & $663(32.0)$ & $392(32.3)$ \\
\hline Sexual and IDU & $537(26)$ & $296(24.5)$ \\
\hline Others & $43(2)$ & $22(1.8)$ \\
\hline HBV co-infection, n (\%) & $182(8.8)$ & $110(9.1)$ \\
\hline HCV co-Infection, n (\%) & $955(46.1)$ & $571(47.1)$ \\
\hline NNRTI rash, n (\%) & $287(14)$ & $55(4.5)$ \\
\hline \multicolumn{3}{|l|}{ Severe reaction, $\mathrm{n}(\%)$} \\
\hline Stevens Johnson syndrome & $19(0.9)$ & $1(0.1)$ \\
\hline Toxic epidermal necrolysis & $1(0.1)$ & $0(0.0)$ \\
\hline Receiving gradual dose of NVP, n (\%) & $1,977(95.5)$ & - \\
\hline
\end{tabular}

HIV: human immunodeficiency virus, IDU: intravenous drug use, HBV: hepatitis B viral, HCV: hepatitis C viral 


\section{Nevirapine-Associated Rash}

In this study, cumulative incidence of NVP-associated rash was $14 \%$ (95\% CI: $12.5-15.5 \%)$. There were 287 patients receiving NVP, either as naïve therapy or substitution drug. Stevens-Johnson Syndrome occurred in $0.9 \%$ of NVP-users and TEN occurred in $0.1 \%$ of them, but no patients died due to these reactions. In multivariate analysis, we found three predictors which were significantly related to NVP-associated rash.
They were female sex (adjusted RR 1.622; 95\% CI: 1.1962.199), baseline CD 4 cell count above 200 cells $/ \mathrm{mm}^{3}$ (adjusted RR 1.387; 95\% CI: 1.041-1.847), and hepatitis C co-infection (adjusted RR 1.507; 95\% CI: 1.138-1.995). Baseline alanin aminotransferase (ALT) level above 1.25 times upper normal limit (ULN) had a tendency to be a good predicting factor (adjusted RR 1.508; 95\% CI: 0.998-2.278) as shown in table 2.

Table 2. Bivariate and Multivariate Analysis of Nevirapine-Associated Rash Predictors (287 cases of 2,170)

\begin{tabular}{|c|c|c|c|c|c|c|}
\hline & \multicolumn{2}{|c|}{ NVP-associated rash } & \multicolumn{2}{|c|}{ Bivariate Analysis } & \multicolumn{2}{|c|}{ Multivariate Analysis } \\
\hline & $\begin{array}{c}\text { Yes } \\
(n=287)\end{array}$ & $\begin{array}{c}\text { No } \\
(\mathrm{n}=1, \mathbf{7 8 4})\end{array}$ & p value & $\mathbf{R R}(95 \% \mathrm{CI})$ & $p$ value & $\operatorname{adjRR}(95 \% \mathrm{CI})$ \\
\hline \multicolumn{7}{|l|}{ Gender, n (\%) } \\
\hline Female & $98(17.0)$ & $478(83.0)$ & 0.010 & $1.346(1.075-1.684)$ & 0.002 & $1.622(1.196-2.199)$ \\
\hline Male & $189(12.6)$ & $1,306(87.4)$ & & & & \\
\hline \multicolumn{7}{|l|}{ Baseline CD4+ count, n (\%) } \\
\hline$>200 \mathrm{cell} / \mathrm{mm}^{3}$ & $86(17.6)$ & $403(82.4)$ & 0.006 & $1.384(1.098-1.744)$ & 0.025 & $1.387(1.041-1.847)$ \\
\hline$<200 \mathrm{cell} / \mathrm{mm}^{3}$ & $201(12.7)$ & $1,381(87.3)$ & & & & \\
\hline \multicolumn{7}{|l|}{ NVP gradual dose escalation, n (\%) } \\
\hline Receiving & $270(13.7)$ & $1,707(86.3)$ & 0.225 & $1.324(0.849-2.065)$ & & \\
\hline Not receiving & $17(18.1)$ & $77(81.9)$ & & & & \\
\hline \multicolumn{7}{|l|}{ Hepatitis C infection, n (\%) } \\
\hline Yes & $145(15.2)$ & $810(84.8)$ & 0.106 & $1.193(0.963-1.479)$ & 0.004 & $1.507(1.138-1.995)$ \\
\hline No & $142(12.7)$ & $974(87.3)$ & & & & \\
\hline \multicolumn{7}{|l|}{ Hepatitis B infection, n (\%) } \\
\hline Yes & $27(14.8)$ & $155(85.2)$ & 0.690 & $1.078(0.748-1.554)$ & & \\
\hline No & $260(13.8)$ & $1,629(86.2)$ & & & & \\
\hline \multicolumn{7}{|l|}{ HIV stage, n (\%) } \\
\hline Stage I-II & $85(15.7)$ & $455(84.3)$ & 0.141 & $1.193(0.944-1.507)$ & & \\
\hline Stage III-IV & $202(13.2)$ & $1,329(86.8)$ & & & & \\
\hline \multicolumn{7}{|l|}{ Baseline AST level, n (\%) } \\
\hline$>1.25 \times \mathrm{ULN}$ & $61(16.2)$ & $316(83.8)$ & 0.149 & $1.213(0.935-1.573)$ & & \\
\hline$<1.25 \times \mathrm{ULN}$ & $226(13.3)$ & $1,468(86.7)$ & & & & \\
\hline \multicolumn{7}{|l|}{ Baseline ALT level, n (\%) } \\
\hline$>1.25 \times \mathrm{ULN}$ & $32(18.7)$ & $139(81.3)$ & 0.055 & $1.394(1.00-1.945)$ & 0.051 & $1.508(0.998-2.278)$ \\
\hline$<1.25 \times \mathrm{ULN}$ & $255(13.4)$ & $1,645(86.6)$ & & & & \\
\hline Body weight, kg, median (min-max) & $53(32-100)$ & $53(25-100)$ & 0.783 & & & \\
\hline
\end{tabular}

RR: Risk Ratio; adj RR: Adjusted Risk Ratio; CI: Confidence Interval; AST: Aspartate Aminotransferase; ALT: Alanine Aminotransferase;ULN: Upper Normal Limit 
Table 3. Bivariate Analysis of Predictors Efavirenz-Associated Rash (55 cases of 1,212)

\begin{tabular}{|c|c|c|c|c|}
\hline \multirow{2}{*}{ Variable } & \multicolumn{2}{|c|}{ EFV-associated rash } & \multicolumn{2}{|c|}{ Bivariate Analysis } \\
\hline & $\begin{array}{c}\text { Yes } \\
(\mathbf{n}=55)\end{array}$ & $\begin{array}{c}\text { No } \\
(n=1,157)\end{array}$ & $p$ value & RR $(95 \%$ CI $)$ \\
\hline \multicolumn{5}{|l|}{ Gender, n (\%) } \\
\hline Female & $17(5.7)$ & $283(94.3)$ & 0.279 & $1.360(0.779-2.373)$ \\
\hline Male & $38(4.2)$ & $874(95.8)$ & & \\
\hline \multicolumn{5}{|l|}{ Baseline CD4+ count, n (\%) } \\
\hline$>200 \mathrm{cell} / \mathrm{mm}^{3}$ & $10(3.8)$ & $250(96.2)$ & 0.545 & $0.814(0.416-1.592)$ \\
\hline$<200 \mathrm{cell} / \mathrm{mm}^{3}$ & $45(4.7)$ & $907(95.3)$ & & \\
\hline \multicolumn{5}{|l|}{ Hepatitis C infection, $\mathrm{n}(\%)$} \\
\hline Yes & $25(4.4)$ & $546(95.6)$ & 0.801 & $0.935(0.557-1.571)$ \\
\hline No & $30(4.7)$ & $611(95.3)$ & & \\
\hline \multicolumn{5}{|l|}{ Hepatitis B infection, $\mathrm{n}(\%)$} \\
\hline Yes & $9(8.2)$ & $101(91.8)$ & 0.054 & $1.960(0.986-3.896)$ \\
\hline No & $46(4.2)$ & $1,056(95.8)$ & & \\
\hline \multicolumn{5}{|l|}{ HIV stage, n (\%) } \\
\hline Stage I-II & $9(4.9)$ & $174(95.1)$ & 0.789 & $1.100(0.548-2.208)$ \\
\hline Stage III-IV & $46(4.5)$ & $983(95.5)$ & & \\
\hline \multicolumn{5}{|l|}{ Baseline AST level, n (\%) } \\
\hline$>1.25 \times \mathrm{ULN}$ & $15(5.4)$ & $261(94.6)$ & 0.415 & $1.272(0.713-2.267)$ \\
\hline$<1.25 \times \mathrm{ULN}$ & $40(4.3)$ & $896(95.7)$ & & \\
\hline \multicolumn{5}{|l|}{ Baseline ALT level, n (\%) } \\
\hline$>1.25 \times \mathrm{ULN}$ & $4(3.9)$ & $98(96.1)$ & 0.755 & $0.854(0.315-2.314)$ \\
\hline$<1.25 \times \mathrm{ULN}$ & $51(4.6)$ & $1,059(95.4)$ & & \\
\hline Body weight, kg, median (min-max) & $50(35-100)$ & $52(24-94)$ & 0.322 & \\
\hline
\end{tabular}

RR: Risk Ratio; adj RR: Adjusted Risk Ratio; CI: Confidence Interval; AST: Aspartate Aminotransferase; ALT: Alanine Aminotransferase; ULN: Upper Normal Limit

\section{Efavirenz-Associated Rash}

The cumulative incidence of EFV-associated rash was $4.5 \%$ (95\% CI 3.4-5.8\%). That was 55 of 1212 subjects receiving EFV, either as naive therapy or substitution regimen. Stevens-Johnson Syndrome incidence was $0.1 \%$ without any mortality while no TEN was occured. There was only one variable (hepatitis B co-infection) that potentially could predict EVP-associated rash $(p<0.25)$ in bivariate analysis and therefore multivariate analysis was not performed. Table 3 showed all variables studied in the bivariate analysis.

\section{Discussion}

This retrospective cohort study involved large number of HIV infected adults in tertiary hospital in Jakarta for decent period of time. The incidence of nevirapine-associated rash in this study was $14 \%$ (95\% CI: 12.5-15.5). This finding confirmed the high incidence of NVP-associated rash among Asian population (2.6-54.4\%) although the recommendation for 14 days escalation dose had been strictly followed. ${ }^{4}$
Reports from African and Caucasian population showed lower incidence (3-10.2\% amongAfrican and 6.5-14.8\% among Caucasian) ${ }^{8,10,11}$ The incidence of EFV-rash from our study was 4.5\% (95\% CI: 3.4-5.8) and similar with other reports from different regions. ${ }^{6}$ A report from Brazil revealed that EFV allergy might involve genetic predisposition. Polymorphism in the interleukin- 10 gene promoter $-1082 \mathrm{G} / \mathrm{A}$ can be related to the occurence of allergic reactions to EFV. ${ }^{12}$ Previous study in French suggest that HLA-DRB1 ${ }^{\star} 01$ allele plays an important roe in succeptibility to NVP and EFV cutaneous reactions. ${ }^{13}$ This theory might be the cause of difference in incidence rate of NNRTI-related rash among different races.

The value of CD4 counts was one of the indicators for HIV patient immunity status. Several studies have reported the increasing tendency for NVP hypersensitivity reaction along with the increase of CD4 lymphocyte counts. ${ }^{3,4,7,9}$ The underlying theory of this phenomenon is unclear but probably involves cytokine of Th1 and Th2, when the balance of these two were disrupted causing abundant amount of Th2 
and therefore enables hypersensitivity reaction to take place. Manosuthi et al. ${ }^{9}$ showed that every increment of 50 cells/ $\mathrm{mL}$ of baseline CD4 lymphocyte counts was associated with 1.2 fold likelihood of developing NVP-associated rash. In this study, we also obtained similar result that CD4 lymphocyte counts of above 200 cell $/ \mathrm{mm}^{3}$ was one of the predictors of NVP-associated rash (adjusted RR $=1.387$; 95\% CI: 1.041 1.847; $p=0.025)$. $^{3,4,7,9}$

Sex-related differences in NVP toxicities have been widely reported. ${ }^{10,14,15}$ In this study, the incidence of NVP-associated rash was 1.62 times higher in females than males. Study by Bersoff-matcha et al. ${ }^{15}$ found a more extreme result. Female sex had 7 times higher risk to develop severe rash associated to NVP use. Several investigations have focused on its pharmacokinetics in an attempt to explain the higher frequency of NVP-associated adverse effects among women. La Porte et al. ${ }^{16}$ and Regazzi et al. ${ }^{17}$ reported that median plasma concentration of NVP was higher in women than in men. Variations in lean body mass and composition that affect NVP distribution might account for these inter-sex difference of NVP plasma levels. ${ }^{17}$ It is also possible that women has better awareness and tend to seek treatment earlier than men and therefore has higher CD4 lymphocyte count at the beginning of treatment.

Most of antiretroviral drug metabolism occurs in the liver. Therefore, transaminase levels at the initiation of ART will affect the outcome in HIV/AIDS patients receiving ART. Elevated (1.25 times upper normal limit) ALT at the beginning of antiretroviral therapy (ART) was shown an associated trend with the occurrence of NVP-associated rash (OR 1.508; 95\% CI: $0.998-2.278 ; p=0.051)$. Several studies also report that the risk of ART-related hypersensitivity reactions in HIV patients with various manifestations would have been increased in patients with higher or above upper normal limit of aspartat aminotransferase (AST) and ALT levels at the beginning of ART. ${ }^{18-20}$ However, study by Sulkowski et al. ${ }^{18}$ suggested that ART-related hypersensitivity was not influenced by higher AST and ALT level, but spesifically because the hepatotoxicity due to hepatitis co-infection. Direct effect from ART and co-infection with hepatitis would worsen liver cells condition. This can be detected from the increase of liver enzymes, which may trigger ART-related hypersensitivity reaction. ${ }^{18-20}$

This study also found that co-infection with hepatitis C (HCV) significantly increased the incidence of NVP-associated rash (OR 1.507; 95\% CI: 1.138-1.995; $p=0.004$ ). The pathogenesis was predicted to involve many factors. Beside immune reconstitution syndrome, HIV co-infection with hepatitis would also worsen the inflammation in the hepatic cells, thus increasing the risk for direct antiretroviral toxicity. ${ }^{18-20}$ This is concurrent with the acquired study subject characteristics i.e. the risk of transmission through IDU, which was quite highand also made the same route of transmission for HIV and HCV.

In this study, less than five percents $(4.5 \%, 95 \%$ CI: $3.4-$ $5.8 \%)$ of EFV-users developed EFV-associated rash, much lower than NVP-associated rash (14\%, 95\% CI: 12.5-15.5\%). A meta-analysis by Schubber et al. ${ }^{21}$ shown that patients using NVP are more likely to experience drug hypersensitivity (OR 2.37, 95\% CI: 1.93-2.9) and severe hypersensitivity reactions (OR 2.4, 95\% CI: 1.9-2.9) compared to patients using EFV.
One of the predicted reasons why the incidence of EFV-associated hypersensitivity is relatively low is because the major manifestation of hypersensitivity reaction to EFV is mainly central nervous system (CNS) involvement. The CNS involvement will stop occurring if the therapy is given at bedtime and therefore any complaints will cease and patients can continue the treatment. ${ }^{6,22}$

Genotype differences might be an important factor that could predict the occurrence of EFV-associated rash. A new report by Rodrigueset et al. ${ }^{12}$ showed that 1082AA genotype was significantly more frequent in EFV allergic patients as compared to non-allergic ones $\left(p=0.019 ; \chi^{2}=5.534 ; \mathrm{OR}=\right.$ 3.625; 95\% CI: 1.210-10.860). The allele IL-10-1082A was also identified more often among EFV-allergic patients in population ( $p=0.009 ; \chi^{2}=6.787$; OR $=3.029$; 95\% CI: 1.290 7.111). ${ }^{12}$ Further study is needed in order to obtain more complete picture about predictors related to the EFV and NVP-associated rash, including the role of genetic factors.

None of baseline predictors analyzed in this study had related with EFV-associated rash, including gender, body weight, CD4 lymphocyte count, hepatitis co-infection, baseline transaminase, and WHO clinical stage. Any clinical risk factor that could be used as predictor of EFV rash would be very useful since other studies failed to find one. Risk factors that have been evaluated in other studies are sex, baseline CD4 lymphocyte count levels, BMI level, viral load, co-infection with hepatitis, history of previous allergic reaction to NVP and concomitant therapy with NVP. ${ }^{6,8,22,23}$

This study had several limitations. First, the study design was cohort retrospective that mainly rely on medical record data therefore any missing values in the medical record will affect the result of the study. Secondly, we did not assess the use of concomitant therapy beside ART, which may had cause hypersensitivity reaction in patients. Last, some possible risk factors such as previous history of drug allergy and baseline viral load number were not included in the present study.

In conclusion, female, baseline CD4 absolute count above 200 cells $/ \mathrm{mm}^{3}$, hepatitis C co-infection and baseline ALT level more than 1.25 times upper normal limit were predictors for nevirapine-associated rash in HIV patients. There was no predictor related to EFV-associated rash. We suggest that more stringent evaluation and monitoring should be carefully done in all HIV/AIDS patients who will receive and on NVP therapy, especially if they have the above predictors.

\section{Conflict of interest}

The authors declare that they have no potential conflict of interest.

\section{Acknowledgements}

We would like to thank all POKDI-rangers and dr. Ira Laurentika.

\section{References}

1. Rajesh R, Vidyasagar S, Johnson S, Singh S, Sharma S. Highly active antiretroviral therapy induced adverse drug reactions in Indian human immunodeficiency virus positive patients. Int J Pharm Sci. 2010;2(2): 84-97. 
2. World Health Organization. Antiretroviral therapy for HIV infection in adults and adolescents: recommendations for a public health approach. Geneva: World Health Organization; 2010.

3. Knobel H, Guelar A, Montero M, Carmona A, Luque S, Berenguer N, et al. Risk of side effects associated with the use of nevirapine in treatment-naive patients, with respect to gender and CD4 cell count. HIV Med. 2008;9:14-8.

4. Kiertiburanakul S, Sungkanuparph S, Charoenyingwattana A, Mahasirimongkol S, Sura T, Chantratita W. Risk factors for nevirapine-associated Rash Among HIV-infected patients with low CD4 cell counts in resource-limited settings. Curr HIV Res. 2008;6:65-9.

5. Blas-Garcia A, Apostolova N, Esplugues JV. Future perspectives in NNRTI-based therapy: bases for understanding their toxicity. In: Tang YW, editor. Recent translational research in HIV/AIDS. London: IntechOpen; 2011. p. 275-94.

6. Manosuthi W, Thongyen S, Chumpathat N, Muangchana K, Sungkanuparph S. Incidence and risk factors of rash associated with efavirenz in HIV-infected patients with preceding nevirapine-associated rash. HIV Med. 2006;7:378-82.

7. Ananworanich J, Moor Z, Siangphoe U, Chan J, Cardiello P, Duncombe $\mathrm{C}$, et al. Incidence and risk factors for rash in Thai patients randomized to regimens with nevirapine, efavirenz or both drugs. AIDS. $2005 ; 19$ : 185-92.

8. Sarfo FS, Sarfo MA, Norman B, Phillips R, Chadwick D. Incidence and determinants of nevirapine and efavirenz-related skin rashes in West Africans: nevirapine's epitaph? PLoS One. 2014;9(4):1-7.

9. Manosuthi W, Sungkanuparph S, Tansuphaswadikul S, Inthong Y, Prasithsirikul W, Chottanapund S, et al. Incidence and risk factors of nevirapine-associated skin rashes among HIV-infected patients with CD4 cell counts $<250$ cells/ $\mu$ L. Int J STD AIDS. 2007;18:782-6.

10. Wit FW, Kesselring AM, Gras L, Richter C, van der Ende ME, Brinkman $\mathrm{K}$, et al. Discontinuation of nevirapine because of hypersensitivity reactions in patients with prior treatment experience, compared with treatment- naive patients: the ATHENA cohort study. Clin Infect Dis. 2008;46:933-40.

11. Jahnke N, Yip B, Phillips E, Gutie S, Lima VD, Hogg RS, et al. Determinants of nevirapine hypersensitivity and its effect on the association between hepatitis $\mathrm{C}$ status and mortality in antiretroviral drug-naive HIV-positive patients. AIDS. 2007;21:1561-8.

12. de Oliveira Rodrigues $R$, de Carvalho PG, de Arruda ÉAG, Rabenhorst SHB, da Silva SFR, Ribeiro IF, et al. Interleukin-10 gene polymorphism $(-1082 \mathrm{G} / \mathrm{A})$ and allergy to efavirenz in patients infected with human immunodeficiency virus. Brazilian J Infect Dis. 2014;18(4):445-8.
13. Vitezica ZG, Milpied B, Lonjou C, Borot N, Ledger TN, Lefebvre A, et al. HLA-DRB $1^{\star} 01$ associated with cutaneous hypersensitivity induced by nevirapine and efavirenz. AIDS. 2008;22:539-44.

14. Peters P, Stringer J, McConnell M, Kiarie J, Ratanasuwan W, Intalapaporn P, et al. Nevirapine-associated hepatotoxicity was not predicted by CD4 count $\geq 250$ cells $/ \mu \mathrm{L}$ among women in Zambia, Thailand and Kenya. HIV Med. 2010;11:650-60.

15. Bersoff-matcha SJ, Miller WC, Aberg JA, van der Horst C, Hamrick HJ, Powderly WG, et al. Sex differences in nevirapine rash. Clin Infect Dis. 2001;32:124-9.

16. la Porte C, van der Ende M, Gyssens I. Gender differences in nevirapine and efavirenz pharmacokinetics. 4th international workshop on clinical pharmacology of HIV therapy,Cannes, 27th-29th March 2003. Abs 10: P 3.1 and Abs 15: P 3.6.

17. Regazzi M, Villani P, Seminari E, Ravasi G, Cusato M, Marubbi F, et al Sex differences in nevirapine disposition in HIV-infected patients. AIDS. 2003; 17:2395-400.

18. Sulkowski MS, Thomas DL, Mehta SH, Chaisson RE, Moore RD Hepatotoxicity associated with nevirapine or efavirenz-containing antiretroviral therapy: role of hepatitis C and B infections. Hepatology. 2002;35:182-9.

19. Taiwo BO. Nevirapine toxicity. Int J STD AIDS. 2006;17:364-70.

20. Kamenan BAT, Abrogoua DP, Djadji ATL, Monnet D. Monitoring of early field hepatic tolerance to NNRTI-based regimens with multiple biochemical parameters in Ivorian HIV-1-infected patients: a pilot study. Pharmacol Pharm. 2012;3:79-89.

21. Shubber Z, Calmy A, Andrieux-Meyer I, Vitoria M, Renaud-Théry F, Shaffer N, et al. Adverse events associated with nevirapine and efavirenz-based first-line antiretroviral therapy. AIDS. 2013;27(9): 1403-12.

22. Kiertiburanakul S, Malathum K, Watcharananan S, Sathapatayavongs B, Sungkanuparph S. Predicting factors for unsuccessful switching from nevirapine to efavirenz in HIV-infected patients who developed nevirapine-associated skin rash. Int J STD AIDS. 2009;20:176-9.

23. Kiertiburanakul S, Sungkanuparph S, Malathum K, Watcharananan S, Sathapatayavongs B, Charoenyingwattana A, et al. A model and risk score for predicting nevirapine-associated rash among HIV-infected patients: in settings of low CD4 cell counts and resource limitation. Open AIDS J. 2009;3:24-30. 